\section{An Evaluation of Acute Hydrogen Sulfide Poisoning in Rats through Serum Metabolomics Based on Gas Chromatography-Mass Spectrometry}

\author{
Meiling Zhang, Mingjie Deng, Jianshe Ma, and \\ Xianqin Wang* \\ Analytical and Testing Center of Wenzhou Medical University; \\ Wenzhou 325035, China.
}

Received December 23, 2013; accepted March 4, 2014

Hydrogen sulfide $\left(\mathrm{H}_{2} \mathrm{~S}\right)$ is the second leading cause of toxinrelated deaths in the operational site. Its main target organs of toxic effects are the central nervous system and respiratory system. In this study, we developed a serum metabonomic method, based on gas chromatography-mass spectrometry (GC/MS), to evaluate the effect of acute poisoning by hydrogen sulfide on rats. Pattern recognition analysis, including both principal component analysis (PCA) and partial least squares-discriminate analysis (PLS-DA), revealed that acute hydrogen sulfide poisoning induced metabolic perturbations. Compared to the control group, the level of urea, glucose, glyceryl stearate in rat serum of the poisoning group increased after two hours, and the level of glucose, docosahexaenoic acid, glyceryl stearate and arachidonic acid in rat serum of the poisoning group increased after $48 \mathrm{~h}$, while the L-valine, galactose, L-tyrosine levels decreased. Our results indicate that metabonomic methods based on GC/MS may be useful to elucidate acute hydrogen sulfide poisoning through the exploration of biomarkers.

Key words metabolomics; GC/MS; acute hydrogen sulfide poisoning

Hydrogen sulfide is a colorless, flammable, and extremely hazardous gas that naturally occurs in hot springs, crude petroleum, and natural gas. The bacterial decomposition of organic materials and human or animal waste also generates hydrogen sulfide.

Forensic toxicology has shown that hydrogen sulfide is one of the most toxic gases. ${ }^{1)}$ It has been reported that if $500 \mathrm{ppm}$ of hydrogen sulfide is in the air, inhalation will rapidly lead to severe poisoning, and that $900 \mathrm{ppm}$ of hydrogen sulfide in the air will cause fatality immediately. ${ }^{2)}$ Accidents have been reported in chemical processing plants, ${ }^{3,4)}$ in sewage disposal facilities, ${ }^{5)}$ and after the ingestion of sulfur products. ${ }^{6)}$ It is difficult to quantify original hydrogen sulfide levels, because the gas produced in these situations is unstable and rapidly metabolized as thiosulfate and sulfate through hepatic oxidation. ${ }^{7)}$ Toxicological analyses of sulfide and thiosulfate, a metabolite of sulfide, in blood and urine of the victims were made after fatal hydrogen sulfide poisoning in the forensic case, using the extractive alkylation technique combined with gas chromatography/mass spectrometry. ${ }^{8,9)}$ In our previous study, "Cocktail" probe drugs approach is used to evaluate the induction or inhibition effects of chronic hydrogen sulfide poisoning on the activities of rats cytochrome $\mathrm{P} 450$ isoforms such as CYP1A2, CYP2C9, CYP2B6 and CYP2D6, as a result

The authors declare no conflict of interest. chronic hydrogen sulfide poisoning could induce the activity of CYP1A2 and CYP2B6 in rats. ${ }^{10)}$

"Metabonomics" has been defined as "the quantitative measurement of the time-related, multi-parametric metabolic response of living systems to pathophysiological stimuli or genetic modification."11) Metabolomics is a scientific study that aims to identify and quantify the levels of all metabolites; it has been applied widely in many areas, such as medicine, biology, and food science. ${ }^{12-15)}$ GC-MS is an analytic method used frequently in metabolomics studies to examine low-molecular-weight metabolites, because GC-MS provides heightened equipment stability and user-friendly tools for data analysis. $^{16,17)}$ This paper evaluates, through the use of $\mathrm{GC} /$ MS-based metabolomics, the effect of acute hydrogen sulfide poisoning on the metabolic profiles of rats.

\section{Experimental}

Chemicals and Reagents $N$-Methyl- $N$-(trimethylsilyl)trifluoroacetamide (MSTFA) and trimethylchlorosilane (TMCS) were purchased from Sigma-Aldrich in Germany. Methylhydroxylamine hydrochloride and pyridine were purchased from Aladdin Industrial, Inc. in China. HPLC-grade acetonitrile and $n$-heptane were purchased from Tedia Reagent Company in the U.S.A.

Instrumentation and Conditions Agilent Technologies, Inc., located in the U.S.A., supplied the Agilent $6890 \mathrm{~N}-5975 \mathrm{~B}$ GC/MS. The column was HP-5MS $(0.25 \mathrm{~mm} \times 30 \mathrm{~m} \times 0.25 \mathrm{~mm}$, Agilent, U.S.A.). The GC oven was initially set at $80^{\circ} \mathrm{C}$ and was kept at this temperature for $5 \mathrm{~min}$. The temperature was then gradually increased to $260^{\circ} \mathrm{C}$ at a rate of $10^{\circ} \mathrm{C} / \mathrm{min}$, and then kept at $260^{\circ} \mathrm{C}$ for $10 \mathrm{~min}$. MS detection was conducted first in EI mode with electron energy of $70 \mathrm{eV}$, then in fullscan mode with $m / z$ of $50-550$, and finally, by splitless mode injection.

Animal Treatment and Sample Collection Male Sprague-Dawley rats, with weights of $250 \pm 20 \mathrm{~g}$, were obtained from the Shanghai SLAC Laboratory Animal Co., Ltd. The animal license number was SCXK (Shanghai) 2012-0005. All fifteen rats were housed at Laboratory Animal Research Center of Wenzhou Medical University under the controlled conditions of a temperature of $22^{\circ} \mathrm{C}$ and a natural light-dark cycle. All experimental procedures were conducted according to the Institutional Animal Care Guidelines and were approved as ethical by the Administration Committee of Experimental Animals at the Laboratory Animal Center of Wenzhou Medical University.

Fifteen male Sprague-Dawley rats were randomly divided into two groups, the control group $(n=8)$ and the acute hydrogen sulfide poisoning group $(n=7)$. To create a model of acute hydrogen sulfide poisoning, the rats were placed in triads in an infected ark that included the hydrogen sulfide detector and then passed into a certain concentration of hydrogen sulfide gases. The rats in the acute hydrogen sulfide poisoning group were exposed to $200 \mathrm{ppm}$ of $\mathrm{H}_{2} \mathrm{~S}$ for 2 and $48 \mathrm{~h}$. The rats in the control group were subjected to similar conditions, but did not experience exposure to $\mathrm{H}_{2} \mathrm{~S}$. The rats in both groups were allowed to eat and drink ad libitum except during the exposure period.

Blood samples of $300 \mu \mathrm{L}$ were drawn from the rats from the control group and acute hydrogen sulfide poisoning group after 2 and $48 \mathrm{~h}$, respectively. The blood samples were placed 
in a water bath of $37^{\circ} \mathrm{C}$ for $0.5 \mathrm{~h}$ and then centrifuged for $10 \mathrm{~min}$ at $8000 \mathrm{rpm}$. The serums were separated and kept at a temperature of $-80^{\circ} \mathrm{C}$ until analysis.

Sample Preparation Two hundred and fifty microliters of acetonitrile was added to $100 \mu \mathrm{L}$ of serum, kept in an icebath for fifteen minutes, and then $10000 \times \boldsymbol{g}$ were centrifuged for $10 \mathrm{~min}$ at $4^{\circ} \mathrm{C}$. One hundred and fifty microliters of the supernatant was transferred to a GC vial and evaporated to dryness under a stream of nitrogen gas. Methoximation was carried out at $70^{\circ} \mathrm{C}$ for $24 \mathrm{~h}$ after $50 \mu \mathrm{L}$ of methylhydroxylamine hydrochloride $(15 \mathrm{mg} / \mathrm{mL}$ in pyridine) was added. Fifty microliters of MSTFA (with $1 \%$ TMCS as the catalyst) was added and kept at $70^{\circ} \mathrm{C}$ for another hour, and then vortexed after adding $150 \mu \mathrm{L} n$-heptane.

Data Analysis The GC/MS data was exported into Microsoft Excel, with the peaks normalized to the total sum of spectrum prior to multivariate analyses. The resulting data was processed through principal component analysis (PCA)

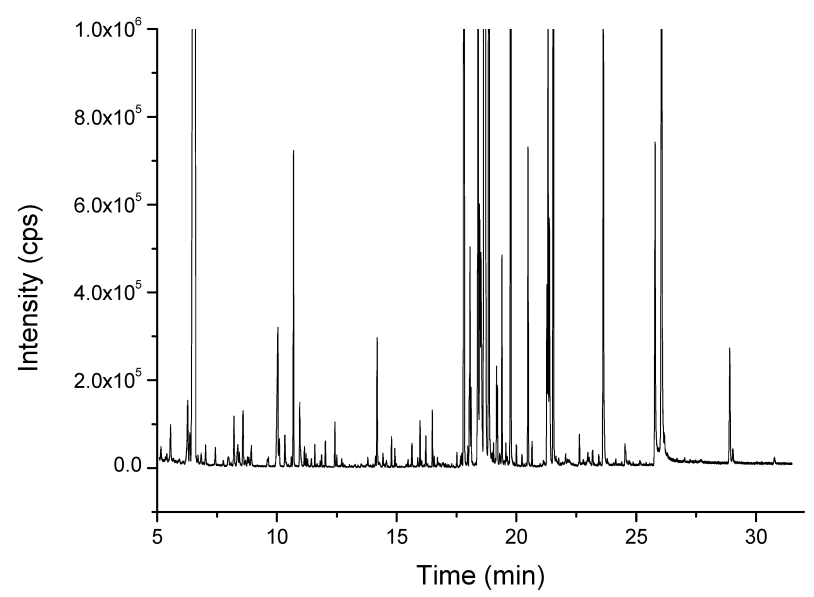

Fig. 1. GC-MS Total Ion Chromatogram of Rat Serum and partial least squares discriminate analysis (PLS-DA) using SIMCA-P 12 software (Umetrics, Umea, Sweden). PLS-DA was used to revealed the differences in serum composition of two different groups, the corresponding loading plots, where each point represents a metabolite, were used to identify which variables contributed to the separation of the samples on the scores plot.

Statistical Analysis Statistical analysis was carried out using SPSS software (Version13.0, SPSS). Independent samples $t$-test was applied in order to detect significant differences in all metabolites between two groups. A $p$ value of $<0.05$ was considered statistically significant.

\section{Results and Discussion}

Analytical Characteristics of Global Profiling Methods Figure 1 provides the typical metabolic profiles of serum acquired through GC-MS technique. Metabolic profile data pretreatment resulted in a final dataset consisting of fortysix metabolic features from GC-MS analyses. The five QC samples first investigated the reproducibility of the metabolic features. The GC-MS analysis showed that more than $70 \%$ of the forty-six metabolic features had a CV\% (coefficient of variance) of no more than $30 \%$. The endogenous metabolites in the serum were identified using the NIST 2005 mass spectrometry database.

Metabolomics Study Principal component analysis of the results of acute hydrogen sulfide poisoning provided an unsatisfactory separation of data between the acute hydrogen sulfide poisoning group and the control group. To improve the classification of the acute hydrogen sulfide poisoning group and control group, we subsequently used a multivariate PLSDA classification method to maximize metabolite variations and to identify the metabolites responsible for such variations.

In order to explore the metabolic profile changes of acute $\mathrm{H}_{2} \mathrm{~S}$ poisoning in rats during different time periods, we com-
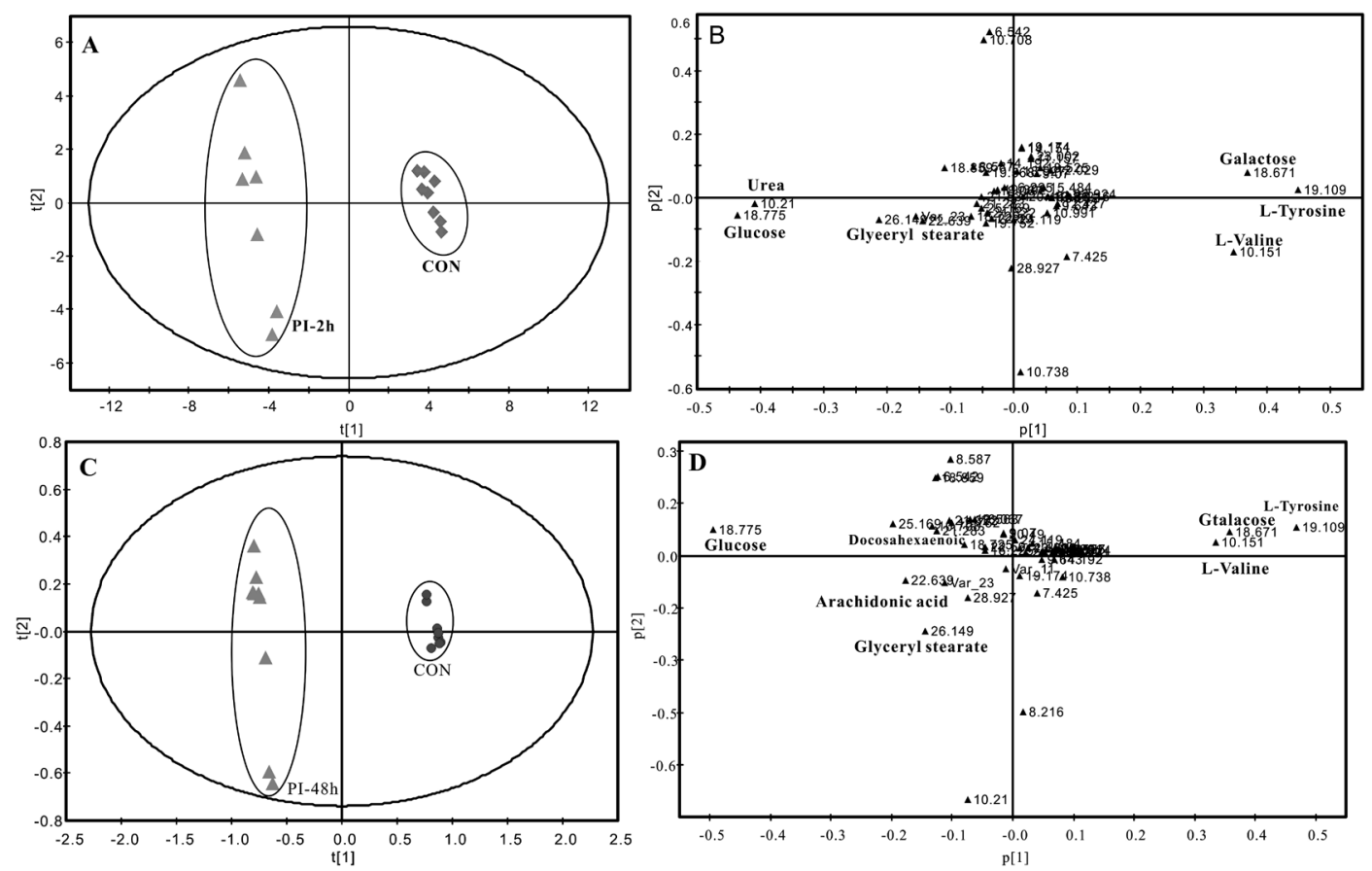

Fig. 2. PLS-DA Score Results of Rat Serum Samples, Acute Hydrogen Sulfide Poisoning after $2 \mathrm{~h}$ Group (PI-2h), Acute Hydrogen Sulfide Poisoning after 48 h Group (PI-48 h), Control Group (CON), B, D Were the Corresponding Load Diagram of A, C 
Table 1. Summary of the Changes in Relative Levels of Metabolites in Rat Serum Indicated by the PLS-DA Loading Plots and Statistical Analysis

\begin{tabular}{|c|c|c|c|c|}
\hline \multirow{2}{*}{ ID } & \multirow{2}{*}{$\begin{array}{l}\text { Retention } \\
\text { time }\end{array}$} & \multirow{2}{*}{ Metabolites compound } & \multicolumn{2}{|c|}{ PI vs. CON } \\
\hline & & & $2 \mathrm{~h}$ & $48 \mathrm{~h}$ \\
\hline 1 & 6.542 & Lactic acid & $\uparrow * *$ & $\uparrow * *$ \\
\hline 2 & 7.425 & L-Alanine & - & $\downarrow *$ \\
\hline 3 & 8.216 & Ethanedioic acid & $\downarrow * *$ & $\downarrow * *$ \\
\hline 4 & 8.587 & Butanoic acid & - & - \\
\hline 5 & 10.151 & L-Valine & $\downarrow * *$ & $\downarrow * *$ \\
\hline 6 & 10.210 & Urea & $\uparrow * *$ & $\uparrow *$ \\
\hline 7 & 10.738 & Phosphate & $\downarrow * *$ & $\downarrow * *$ \\
\hline 8 & 14.192 & Pyrrolidone carboxylic acid & - & $\downarrow * *$ \\
\hline 9 & 18.671 & Galactose & $\downarrow * *$ & $\downarrow * *$ \\
\hline 10 & 18.775 & D-Glucose & $\uparrow * *$ & $\uparrow * *$ \\
\hline 11 & 19.109 & L-Tyrosine & $\downarrow * *$ & $\downarrow * *$ \\
\hline 12 & 19.174 & Ribose & - & $\uparrow * *$ \\
\hline 13 & 19.568 & Palmitelaidic acid & - & - \\
\hline 14 & 19.752 & Hexadecanoic acid & $\downarrow *$ & - \\
\hline 15 & 20.491 & Myo-inositol & - & - \\
\hline 16 & 21.282 & 9,12-Octadecadienoic acid & $\uparrow * *$ & $\uparrow * *$ \\
\hline 17 & 21.322 & Oleic acid & $\uparrow * *$ & $\uparrow * *$ \\
\hline 18 & 22.639 & Arachidonic acid & $\uparrow * *$ & $\uparrow * *$ \\
\hline 19 & 25.169 & Docosahexaenoic acid & $\uparrow *$ & $\uparrow * *$ \\
\hline 20 & 26.149 & Glyceryl stearate & $\uparrow * *$ & $\uparrow *$ \\
\hline 21 & 28.927 & Cholesterol & - & $\uparrow * *$ \\
\hline
\end{tabular}

Marks indicate the direction of the change, i.e., $\downarrow$ for decrease, $\uparrow$ for increase, for no change. ${ }^{*} p<0.05$ and ${ }^{* *} p<0.01$, as indicated by the statistical analysis $t$-test.

pared the GC-MS spectrum of PLS-DA of the rats in the acute $\mathrm{H}_{2} \mathrm{~S}$ poisoning group after $2 \mathrm{~h}$ and after $48 \mathrm{~h}$ with the rats in the control group (Fig. 2). Figure 2A PLS-DA score chart $(\mathrm{R} 2 \mathrm{X}=0.715, \mathrm{Q} 2 \mathrm{Y}=0.998)$ showed that the first principal components of the rats in the acute $\mathrm{H}_{2} \mathrm{~S}$ poisoning group after $2 \mathrm{~h}$ were distinguished from the rats in the control group. The corresponding load diagram (Fig. 2B) showed the major metabolites that separated PI-2h from control group were urea, glucose, glyceryl stearate the L-valine, galactose, L-tyrosine.

As demonstrated in Fig. 2C, the PLS-DA score $(\mathrm{R} 2 \mathrm{X}=0.73$, $\mathrm{Q} 2 \mathrm{Y}=0.995)$ showed that the acute $\mathrm{H}_{2} \mathrm{~S}$ poisoning after $48 \mathrm{~h}$ group and the control group were separated. The corresponding load diagram (Fig. 2D) better distinguishes the metabolites of the two groups. Figure 2D showed the major metabolites that separated PI-2h from control group were glucose, docosahexaenoic acid, glyceryl stearate, arachidonic acid L-valine, galactose, L-tyrosine.

Changes in Metabolite The changes of metabolites between poisoning groups and their control group were shown in Table 1. Compared to the control group, the level of urea, glucose, glyceryl stearate in the rat serum of the acute $\mathrm{H}_{2} \mathrm{~S}$ poisoning group increased after two hours, while the L-valine, galactose, L-tyrosine level decreased. And the level of glucose, docosahexaenoic acid, glyceryl stearate and arachidonic acid in rat serum of the acute $\mathrm{H}_{2} \mathrm{~S}$ poisoning group increased after forty-eight hours group, while the L-valine, galactose, Ltyrosine levels decreased.

In previous study, it should need to pay close attention to changes in the plasma concentration to avoid drug interactions that may occur, when take drugs by intravenous that metabolized through CYP1A2 and CYP2B6 enzyme after chronic hydrogen sulfide poisoning. ${ }^{10)}$ In this study, we found glucose, glyceryl stearate, L-valine, galactose, L-tyrosine were in different level between acute $\mathrm{H}_{2} \mathrm{~S}$ poisoning and control group. These finding may be useful for new evidences in forensic case.

\section{Conclusion}

These biomarkers (glucose, glyceryl stearate, L-valine, galactose, L-tyrosine) were the additional evidence that supports already-known evidence sulfide and thiosulfate, a metabolite of sulfide. We demonstrated that metabonomic methods based on GC/MS could provide a useful tool for exploring biomarkers to elucidate poisoning discovered in forensic toxicology.

Acknowledgments This study was supported by Grants from the National Natural Science Foundation of China, No. 81102297; the Science and Technology Committee of Shanghai Municipality, China, No. KF1101.

\section{References}

1) Maebashi K., Iwadate K., Sakai K., Takatsu A., Fukui K., Aoyagi M., Ochiai E., Nagai T., Forensic Sci. Int., 207, 91-95 (2011).

2) Tatsuno Y., Adachi J., Mizoi Y., Fujiwara S., Nakanishi K., Taniguchi T., Yokoi S., Shimizu S., Nippon Hoigaku Zasshi, 40, 308-315 (1986).

3) Gabbay D. S., De Roos F., Perrone J., J. Emerg. Med., 20, 141-144 (2001).

4) Tanaka S., Fujimoto S., Tamagaki Y., Wakayama K., Shimada K., Yoshikawa J., Am. J. Emerg. Med., 17, 427-429 (1999).

5) Snyder J. W., Safir E. F., Summerville G. P., Middleberg R. A., Am. J. Emerg. Med., 13, 199-203 (1995).

6) Kage S., Nagata T., Kudo K., J. Anal. Toxicol., 15, 148-150 (1991).

7) Dorman D. C., Moulin F. J., McManus B. E., Mahle K. C., James R. A., Struve M. F., Toxicol. Sci., 65, 18-25 (2002).

8) Kage S., Ikeda H., Ikeda N., Tsujita A., Kudo K., Leg. Med., 6, 182-186 (2004).

9) Maebashi K., Iwadate K., Sakai K., Takatsu A., Fukui K., Aoyagi M., Ochiai E., Nagai T., Forensic Sci. Int., 207, 91-95 (2011).

10) Wang X., Chen X., Chen M., Hu G., Ma J., Pan J., Hu L., Lin G., Biol. Pharm. Bull., 36, 1627-1633 (2013).

11) Nicholson J. K., Connelly J., Lindon J. C., Holmes E., Nat. Rev. Drug Discov., 1, 153-161 (2002).

12) Clasquin M. F., Melamud E., Singer A., Gooding J. R., Xu X., Dong A., Cui H., Campagna S. R., Savchenko A., Yakunin A. F., Rabinowitz J. D., Caudy A. A., Cell, 145, 969-980 (2011).

13) Nishiumi S., Kobayashi T., Ikeda A., Yoshie T., Kibi M., Izumi Y., Okuno T., Hayashi N., Kawano S., Takenawa T., Azuma T., Yoshida M., PLoS ONE, 7, e40459 (2012).

14) Sreekumar A., Poisson L. M., Rajendiran T. M., Khan A. P., Cao Q., Yu J., Laxman B., Mehra R., Lonigro R. J., Li Y., Nyati M. K., Ahsan A., Kalyana-Sundaram S., Han B., Cao X., Byun J., Omenn G. S., Ghosh D., Pennathur S., Alexander D. C., Berger A., Shuster J. R., Wei J. T., Varambally S., Beecher C., Chinnaiyan A. M., Nature (London), 457, 910-914 (2009).

15) Vrhovsek U., Masuero D., Gasperotti M., Franceschi P., Caputi L., Viola R., Mattivi F., J. Agric. Food Chem., 60, 8831-8840 (2012).

16) Hiller K., Hangebrauk J., Jager C., Spura J., Schreiber K., Schomburg D., Anal. Chem., 81, 3429-3439 (2009).

17) Tsugawa H., Tsujimoto Y., Arita M., Bamba T., Fukusaki E., BMC Bioinformatics, 12, 131-143 (2011). 